\title{
VALORACIÓN DE EMPRESAS POR EL MÉTODO DE LOS RENDIMIENTOS FUTUROS
}

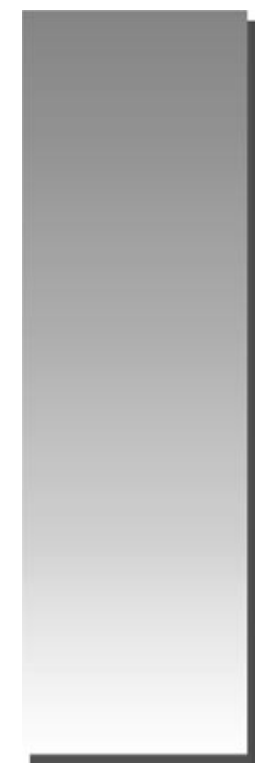

Alejandro Narváez Liceras*

E-mail:narvaez1002@gmail.com

"No hay nada tan peligroso como la búsqueda de una política de inversión racional en un mundo irracional".

John MAYnARd Keynes

\section{RESUMEN}

Muchos académicos y profesionales del mundo de los negocios han dicho, más de una vez, que la valoración de empresas es en gran medida un arte para manejar los números según unos objetivos definidos. Sin duda algo puede haber de cierto en esta afirmación; empero, esta actividad tiene mucho de conocimiento profundo de las dificultades y problemas asociados para asignarle el valor "real" a una empresa en marcha. Este artículo tiene como objeto examinar diversos aspectos de la valoración de empresas aplicando el método de los rendimientos futuros. Se explican algunas de las razones por las cuales se elige este método, y se aborda la forma cómo se cuantifican las variables que intervienen.

Palabras clave: Valoración de empresas, flujos de caja, tasa de actualización, coeficiente beta, costo medio ponderado de capital.

\section{ABSTRACT}

Many educators and professionals of the business world have said, more than one time, that the appraisal of businesses is in great measure an art to handle the numbers according to some definite objectives. Without doubt, something can should certain in this affirmation however, this activity has a lot of thorough knowledge of the difficulties and associated problems to assign him the value "real" to a going company. This article has as object to examine diverse aspects of the appraisal of businesses applying the method of the future performances. They are explained some of the reasons by which this method is chosen, and the form is undertaken how the variables are quantified.

Key words: Appraisal of business, cash flows, discount rate, beta coefficient, medium cost praised of capital.

* Doctor en Ciencias Económicas. Magíster en Finanzas. Consultor nacional e internacional. Profesor Asociado de la Facultad de Ciencias Administrativas de la UNMSM 


\section{CONSIDERACIONES PREVIAS}

Actualmente, la valoración de empresas no sólo se ve como una actividad de aplicación puntual al servicio de una eventual operación de adquisición o fusión; es también una excelente herramienta de planificación y gestión de negocios a medio y largo plazo. Por ello, conocer cuánto vale una empresa en marcha, en un momento concreto del tiempo, es una de las cuestiones que importan mucho, principalmente a quienes están ligados al mundo de los negocios.

En el proceso de valoración de una empresa o activo es necesario que, previamente a la adopción del criterio o método a seguir, se conozca la finalidad de la valoración, la tipología, características y situación real en la que se encuentra la empresa objeto de valoración.

Para valorar de manera correcta a una empresa hay que conocerla previamente, analizando toda la información disponible acerca del sector en el que compite, su posición relativa (fortalezas y debilidades), sus planes futuros, etc., que serán determinantes para adoptar el criterio o los criterios de valoración que se consideran pertinentes; es decir, hacer lo que habitualmente se conoce como due diligence.

Siempre es recomendable conocer y utilizar una metodología de valoración para que, mediante un proceso lógico de análisis de la situación de la empresa, consolidación de la información obtenida y utilización del criterio de valoración adecuada, se desarrolle un correcto informe del trabajo.

Existen inconvenientes en casi todos los métodos de valoración propuestos por académicos y profesionales de negocios que hacen que, en la práctica, rara vez sean aplicables tal y como figuran en los manuales y revistas especializadas o que, en su aplicación, lleven a decisiones erróneas. En general, los métodos de valoración teóricamente más idóneos son los que tienen mayor dificultad en su aplicación y viceversa, principalmente debido a la necesidad y costo de hacer estimaciones fiables. No obstante, el método del Flujo de Caja Descontado (FCD o Free Cash Flow), parece ser el más perfeccionado y robusto que existe, razón por la cual en este trabajo se presenta una descripción sintética de la metodología que debe seguirse para su correcta aplicación.

\section{MÉTODO DEL RENDIMIENTO FUTURO ESPERADO.}

El fin para el que se encarga la valoración condiciona el método a seguir, por lo que toda valoración debe comenzar con una reunión entre el solicitante de la valoración y el valorador (tasador), en la que queden establecidos -con la mayor claridad posible- los fines que se persiguen y se llegue a un claro entendimiento del procedimiento a seguir.

Cuando hablamos de valoración de empresas, hablamos de valorar la empresa en su conjunto como un negocio en marcha. Hay que distinguir esta valoración de la simple valoración de activos fijos individualmente, aunque éstos, además, deben ser estimados cuando se valora la empresa como una unidad económica.

En teoría existen numerosos métodos de valoración de empresas, sin embargo, suelen agruparse principalmente en tres tipos: métodos basados en el análisis de los estados financieros, métodos basados en la cotización bursátil y métodos basados en la actualización de los flujos o rendimientos futuros esperados.

En la práctica, el método basado en la actualización de los rendimientos futuros esperados es el más utilizado para la valoración de empresas por su capacidad analítica y rigor, si bien su aplicación no está exenta de dificultades. Sus principales ventajas son posibilitar el examen de los factores que crean o destruyen valor para la empresa, y reconocer de forma explícita el diferente valor temporal del flujo de caja de la empresa.

El método basado en la actualización de los rendimientos futuros parte del principio de que, para el comparador de una empresa, por ejemplo, el pasado tiene poca importancia, y que lo que le interesa conocer es el potencial de generar rendimientos o flujos de caja en el futuro. Esto quiere decir que hay que hacer una previsión de los rendimientos de la empresa, y más exactamente, elaborar estados de ganancias y pérdidas y balances proyectados de los años futuros. Como el método se basa en predicciones, los resultados pueden ser erróneos si las predicciones son equivocadas.

Con el fin de evitar errores es imprescindible comenzar la valoración con un análisis exhaustivo del sector y de la empresa revisando, en colaboración con el equipo directivo, la fuerza relativa de 
los proveedores, clientes, competidores actuales y futuros, planes futuros, sustitutivos potenciales de productos o servicios de la empresa, expectativas de crecimiento del sector, sus factores críticos de éxito, composición del accionariado, proyectos $\mathrm{I}+\mathrm{D}+\mathrm{i}$, posibles sinergias ${ }^{1}$ que pueda representar para el comparador, etc. y en relación a estos factores cuáles son las fortalezas y debilidades de la empresa valorada.

Un nuevo paso necesario es la valoración del pasivo de la empresa. El momento más importante es el establecimiento de cuáles son las alternativas posibles y razonables para el futuro de la empresa. Cuando se establezcan no hay que olvidar que una de estas alternativas es siempre la liquidación del negocio. Cada una de las alternativas debe ser valorada por el método de actualización de los flujos de caja y, lógicamente, elegiremos entre las posibles a aquella que produzca mayores rendimientos. El valor que resulte de analizar esta alternativa será el valor de la empresa.

\section{Cálculo de los Flujos de Caja Futuros}

Si suponemos que se dispone de información primaria y directa proporcionada por la dirección de la empresa, se tratará de calcular, bajo determinados supuestos de comportamiento del entorno, sector y empresa, cuales serían los distintos flujos de entrada y salida de caja: operativos, de inversión y financieros. Se trata, en definitiva, de efectuar proyecciones sobre inversiones en el horizonte de tiempo fijado, así como determinar la forma cómo deben financiarse dichas inversiones, etc.; en suma, se trata de formular estados financieros proyectados (estados de ganancias y pérdidas y balances).

Según lo dicho, los flujos de caja futuros (FCF) que se toman para la valoración de un año cualquiera se pueden definir como sigue:

$F C O=B N+D p+G F n i$

Luego,

$$
F C F=F C O-I a f-I c t+V R a f b+V R c t
$$

Donde:

FCO : Flujo de Caja Operativo

$B N$ : Beneficio neto después de impuestos.

$D p$ : Depreciación (amortización).
GFni: Gastos financieros netos de impuesto.

Iaf: Inversiones en activo fijo.

Ict: Inversiones en capital de trabajo.

$V R a f b$ :Valor de recuperación de activos fijos dados de baja.

VRct: Valor de recuperación del capital de trabajo.

El valor total de la empresa o negocio vendría determinado, en el momento presente, por el valor actual de los flujos de caja neto esperados $\left(V A F C F_{E}\right)$ más el valor residual actualizado según la tasa de descuento considerada relevante para el caso. Es decir:

$$
\operatorname{VAFCF}_{E}=\sum_{1=1}^{n} \frac{F C F_{t}}{(1+C M P C)^{t}}+\frac{R_{n}}{(1+C M P C)^{n}}
$$

lo que es lo mismo,

$V A F C F_{E}=\frac{F C F_{1}}{(1+C M P C)}+\frac{F C F_{2}}{(1+C M P C)^{2}}+\ldots .+\frac{F C F_{n}+K_{n}}{(1+C M P C)^{n}}$

Donde:

$\mathrm{n}=$ número de años $(1,2, \ldots \mathrm{n})$.

$F C F_{t}=$ Flujo de Caja Futuro en el momento t.

$\mathrm{CMPC}=$ Costo Medio Ponderado del Capital (en inglés, Weighted Average Cost of Capital o WACC).

$\mathrm{VR}_{\mathrm{n}}=$ Valor Residual de los FCF esperados después del periodo con previsiones explícitas del año $n$.

\section{Estimación de la tasa de actualización}

Como puede verse en el Gráfico №1, existen distintos métodos para estimar la tasa de actualización o descuento; no obstante, en la práctica, se considera relevante utilizar la tasa correspondiente al coste medio ponderado del capital de la empresa (CMPC). Para su cálculo hay que definir la combinación de recursos ajenos o deuda y recursos propios que se juzgue apropiada y conocer el costo de cada una de ellas, como sigue:

$$
C M P C=W_{R A} * K_{R A}(1-t)+W_{R P} * K_{R P}
$$

$\mathrm{O}$ lo que es igual:

$$
C M P C=K_{R A}(1-t) * \frac{R A}{R A+R A}+K_{R P} * \frac{R P}{R A+R A}
$$

\footnotetext{
1 Cuando se logra incrementar el valor de mercado de la empresa para sus accionistas o para los stakeholders, es decir, cuando el resultado es superior a la suma de las partes, se habrá producido una sinergia positiva, de lo contrario habrá surgido una sinergia negativa.
} 
Donde:

$\mathrm{K}_{R A}=$ Costo de los recursos ajenos

$\mathrm{t}=$ Tasa del impuesto a la renta sobre beneficios.

$\mathrm{W}_{R A}=$ Ponderación de los Recursos Ajenos en la estructura financiera esperado.

KRP $=$ Costo de los Recursos Propios

$\mathrm{W}_{R P}=$ Ponderación de los Recursos Propios en

la estructura financiera esperado o deseado.

Gráfico N. ${ }^{\circ}$ 1. Métodos más utilizados para el cálculo de la tasa de actualización.

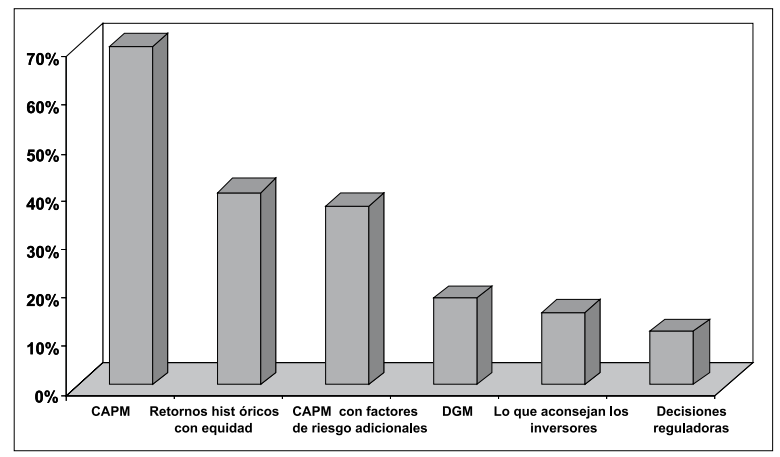

Fuente: Graham and Harvey. The theory and practice of corporate finance: evidence from the field. Journal of Financial Economics, May 2001.

\section{Estimación del costo de los recursos propios}

El costo de los Recursos Propios $\left(K_{r p}\right)$ es más complicado de estimar. En la práctica resulta de aplicar el Modelo de Equilibrio de Activos Financieros (Capital Asset Pricing Model o CAPM) que vendría a ser la suma de una tasa libre de riesgo (bonos del Estado a largo plazo) más una prima de riesgo $\left(P_{r}\right)$ multiplicado por un coeficiente beta. ${ }^{2}$ :

$K_{R P}=R_{f}+P_{f} * \beta$

$\mathrm{O}$ lo que es lo mismo,

$\mathrm{K}_{R P}=\mathrm{R}_{f}+\left(\mathrm{R} \mathrm{m}-\mathrm{R}_{f}\right) * \beta\left(3^{`}\right)$

Donde:

$\mathrm{P}_{r}=$ Prima de riesgo del mercado $\left(\mathrm{R}_{m}-\mathrm{R}_{f}\right)$.

$\mathrm{R}_{f}=$ Rentabilidad de Activos Financieros Libre de riesgo (Bonos del Estado de Largo Plazo).

$\beta=$ Coeficiente beta o riesgo sistemático de las acciones de la empresa ${ }^{4}$

$\mathrm{R}_{\mathrm{m}}=$ Rentabilidad media del mercado de renta variable a largo plazo.

$\mathrm{R}_{\mathrm{m}}-\mathrm{R}_{f}=$ Prima de Riesgo del mercado de renta variable.

En el país no se tiene el coeficiente beta sectorial debido a que el mercado de valores de Lima es pequeño y cotizan en ella pocas empresas, por lo que es práctica habitual recurrir a los betas de las empresas americanas, principalmente. El Gráfico №2 muestra betas de empresas americanas por sectores.

El método del flujo de caja descontado (Free Cash Flow) incorpora un criterio de mercado que es el costo de oportunidad del capital que los inversores exigen para proyectos de riesgo similar, obligando a considerar el impacto a largo plazo de las decisiones empresariales sobre la capitalización bursátil de la sociedad y de la riqueza de sus accionistas.

Gráfico №2. Beta de las empresas de Estados Unidos por sectores

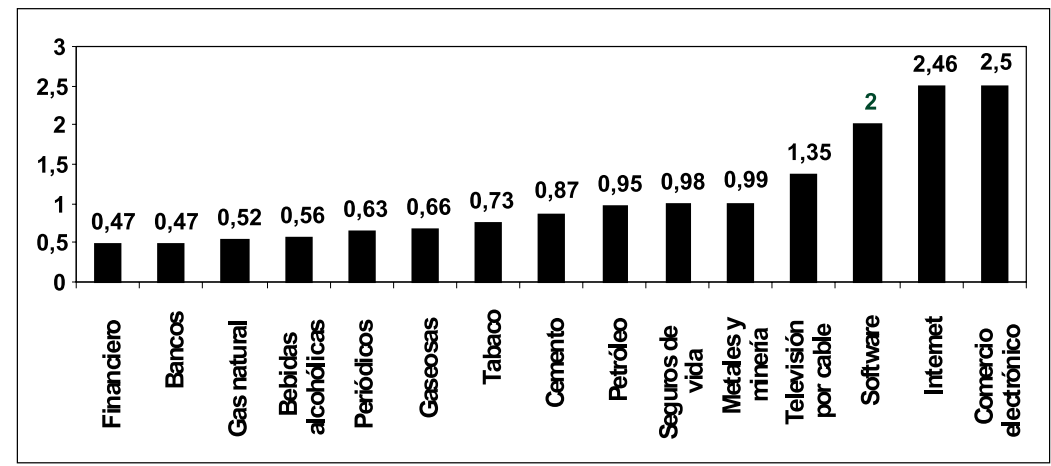

Fuente: www.damodaran.com

2 El modelo de equilibrio de activos financieros (CAPM) es una técnica de valoración de activos que relaciona el riesgo y la rentabilidad esperada. Permite determinar cuál es la cartera de renta fija y acciones que proporciona la mayor rentabilidad esperada asumiendo un riesgo dado.

3 El modelo CAPM fue desarrollado inicialmente por el Premio Nóbel W. Sharpe (1964), seguido por J. Lintner (1965) y posteriormente por el Premio Nóbel R. Merton (1990).

4 La beta de una acción mide el riesgo incremental que aporta una acción a una cartera de valores diversificada. Este riesgo, denominado riesgo sistemático o riesgo de mercado, es el que no se puede eliminar al formar una cartera o portafolio de inversión diversificado. 
Desde este enfoque, el valor de la empresa se considera en función de los aspectos fundamentales: la contribución positiva o negativa de cada una de las estrategias de inversión o de financiación al flujo de caja de la empresa, y su incidencia sobre el costo medio ponderado del capital de la compañía. En definitiva, se trata de estimar la repercusión de estrategias empresariales alternativas sobre el valor de mercado de la sociedad, determinado básicamente por su evolución en términos de flujos de caja esperados y por los niveles de riesgo operativo y financiero que impliquen cada uno de los proyectos. Un proyecto de inversión puede aumentar la cifra de ingresos e incluso el beneficio contable de la sociedad, pero sólo aumentará su valor de mercado a largo plazo si los inversores y accionistas perciben que la tasa de rendimiento interno de las inversiones es superior al costo de oportunidad del capital invertido en negocios de riesgo equivalente.

\section{Estimación del valor residual de la empresa}

Se trata del valor que se espera que tuviera la empresa una vez concluido el horizonte temporal estimado. No existe un único método para estimar este valor pero sí se conoce que dicho valor depende de los supuesto manejados a priori para el cálculo de los flujos de caja, y es un dato clave para establecer el valor final del negocio. Luego, el $\mathrm{VR}_{f}$ resulta de la siguiente expresión:

Donde:

$$
V R_{n}=\frac{\left(\mathrm{FCF}_{n}\right)(1+\mathrm{g})}{C M P C-G}
$$

$\mathrm{VR}_{n}=$ Valor residual calculado del último año del período estimado.

$\mathrm{g}=$ Tasa esperada de crecimiento perpetuo de Free Cash Flow
Cabe señalar que la utilización del método de valoración del flujo de caja descontado no debe ser nunca mecánica siendo esencial, por tanto, realizar un análisis de sensibilidad de los resultados que refleje los elementos de riesgo e incertidumbre que están implícitos en la mayoría de las decisiones empresariales.

Considerar la probabilidad de comportamientos distintos en las variables clave, señalando su impacto sobre la valoración, permitirá alcanzar una mejor comprensión de los riesgos del proyecto. La utilización de escenarios alternativos contribuirá también a identificar los rangos de valores más probables, enriqueciendo la toma de decisiones que finalmente se reflejará en el precio de oferta por la compañía.

A continuación se presentan los resultados de algunos casos peruanos de valoración de empresas privadas y públicas, aplicando el método de flujo de caja de descontado.

\section{ESTRATEGIA DEL PRECIO DE OFERTA}

Es importante diferenciar entre la posición compradora y la posición vendedora, puesto que las motivaciones y los objetivos son totalmente diferentes (incrementar su cuota de mercado versus consolidar su patrimonio). En cada caso, un análisis exhaustivo previo a la operación y una definición clara de los objetivos perseguidos por los compradores, son claves no sólo para el éxito de la operación, sino también para el éxito de la integración y el control posterior.

Pese a la dimensión cuantitativa del método expuesto, se suele decir que la valoración de una empresa tiene más de arte que de ciencia exacta. Incluso el método que en nuestra opinión presenta más ventajas, al basarse en los flujos netos de

Cuadro №1. Casos de empresas peruanas valorados con el método del FCD (en mill. de US\$)

\begin{tabular}{|l|l|r|r|}
\hline Sector & Empresa & Valor Patrimonial & Rango de Valorización \\
\hline Privado & UCPBJ & 2,500 & 2,200 a $-2,800$ \\
\hline & Cervesur & 414 & 370 a 460 \\
\hline & San Juan & 208 & 185 a 234 \\
\hline & Industria de Envases & 21 & 20 a 23 \\
\hline & Cia Minera La Poderosa & 128 & 119 a 137 \\
\hline Público & Solgas & 16 & nd \\
\hline & Hierro Perú & 195 & nd \\
\hline & Pesca Perú & 22 & nd \\
\hline
\end{tabular}

Fuentes: ENFOCA (2006) y Comisión Investigadora de Delitos Económicos y Financieros del Congreso de la República (2001). 
caja como fuente del valor de la empresa, podrá arrojar tantos valores como hipótesis sobre el comportamiento de las variables básicas se deseen utilizar. Ello hace tanto más necesario identificar, mediante el análisis de sensibilidad, aquellos supuestos como ventas, gastos operativos, inversiones en activos fijos, depreciación, horizonte temporal, capital de trabajo, valor residual, etc., que tienen mayor impacto en la valoración final, verificando su grado de realismo en función de las tendencias observadas hasta el presente.

En las fusiones o adquisiciones, tanto el potencial comparador como el vendedor, tendrán diferentes percepciones sobre el rango de valores adecuados a las características de la empresa. El comprador que aspira al control de la empresa objetivo fundará su valoración en un plan de empresa diferenciado, con distintos supuestos operativos y financieros. La toma en consideración de las posibles sinergias tampoco será coincidente, provocando discrepancias en el precio máximo aceptable para cada una de las partes, determinado por el valor actual de los cash flow neto esperados una vez tomado el control de la empresa y aprovechadas todas las sinergias que para el comprador pudieran existir. Estos aspectos resultan de especial interés en los casos de empresas públicas que son privatizadas. Aquí surgirán diferencias en el valor que la empresa representa para distintos inversores como consecuencia de los supuestos que manejan y las tasas de descuento aplicables al proyecto, pudiendo existir disparidades marcadas en las primas por riesgo aplicadas.

En la estrategia de fijación del precio de comparar ventas para garantizar el éxito de la operación, debe existir una diferencia entre el precio mínimo o suelo por el que el vendedor está dispuesto a vender y el precio máximo por el que el comprador está dispuesto a comprar, configurándose de este modo el área de negociación de las partes.

Son los intereses encontrados que se dan cita en las adquisiciones de empresas los que, en definitiva, suelen hacer aconsejable para las partes el disponer de una fairness opinion (opinión clara de los asesores financieros) frente a posibles reclamaciones, por que, para decirlo con palabras de Tomás de Aquino, "el justo precio de las cosas no está determinado con exactitud, sino que consiste en una cierta estimación".

\section{CONCLUSIONES}

1. La valoración de empresas se han convertido en una importante herramienta de planificación y gestión de los negocios; por ello, ya no se le ve como un medio de aplicación puntual en operaciones de adquisición o fusión de negocios.

2. La valoración de la empresa, en una operación de fusión o adquisición, ha de ser fruto de un riguroso trabajo previo conocido como due diligence y que consiste en el análisis y verificación de la información disponible sobre la empresa.

3. El método del Flujo de Caja Descontado resulta ser el más fiable y, consecuentemente, el más utilizado, pues se basa en la capacidad de generar ingresos futuros por un negocio, independientemente que sea privado o público. No obstante, sería recomendable complementarla con un análisis comparativo basado en multiplicadores de empresas similares.

4. Pese a la dimensión cuantitativa del método expuesto, la valoración de una empresa tiene más de arte que de ciencia exacta. Incluso el método que, en nuestra opinión, presenta más ventajas al basarse en los flujos netos de caja como fuente del valor de la empresa, podrá arrojar tantos valores como hipótesis sobre el comportamiento de las variables básicas se deseen utilizar.

\section{BIBLIOGRAFÍA}

Caballero, V. (1998). Manual de Valoración de Empresas. Madrid, Díaz de Santos.

Damodaran, A. (2008). www.damodaran.com on line.

Damodaran, A. (1994). Damodaran on valuatation. New York, John Wily and Sons.

ENFOCA (2006). Informe de valoración de acciones con derecho a voto para la OPA de UCPBJ y subsidiarias.

Fernández, P. (1999). Valoración de Empresas. Barcelona, Gestión 2000.

Garicano, T. (2005). "El descuento de flujos de caja y la valoración por múltiplos”. En: HD Finanzas y Contabilidad. Núm. 59, pp.13-21.

Merton. R. (1973). "An Intertemporal Capital Asset Pricing Model”. En: Econometrica, vol. 41, num. 5, pp. 867-887.

Narváez, A. (1998). "Valoración de empresas: herramienta de gestión”. En: Diario Gestión. 12 de mayo de 1998. 